absolutely necessary, and then only for as short a time as possible, and should never interfere with passive and active movements. I am writing now mainly of the upper extremity, but I am not by any means excluding the lower. Massage is a most excellent adjunct-far and away the best-to active movement. I lay but little stress on electricity or baths.

In war surgery the future usefulness of the wounded must be borne in mind, and in that relation the order of importance is-muscles, joints, and bones; not bones, joints, muscles. If a demand is made upon nature without unclue pain, and carefully advancing, nature will always respond. Disuse, active and passive, means malnutrition of the part. Muscles atrophy, bones atrophy, blood vessels atrophy, pus tends to track along the intermuscular planes; passive and active movement help to drive the pus out.

When passive or active movements are begun early, pain is the danger signal that shows harm is being done, and the movements should be modified temporarily. When after prolonged immobilization adhesions have formed, however, pain has to be endured while the adhesions are being stretched or broken. I am not at all surprised, therefore, that there has been an insistent demand for the services of bonesetters, who overcome the adhesions which have been brought about by well meaning surgeons. Take, for example, the case of a man shot through the biceps. His forearm was placed at a right angle and kept in that position for weeks; he was then unable to extend the arm, and after many long weeks of massage recovery was incomplete. In the case of wounds through the forearm where the bones and nerves have escaped, and treated with splints for prolonged periods, it is found that when movements of a passive and active nature are attempted the patient is unable to carry them out owing to the formation of fibrous tissue during the period of immobilization. The most marked results from muscles involved are found in the hamstring muscles and the calf. How familiar is the sight of convalescent soldiers limping about the streets with bent, knees and on their toes! The majority of these will never straighten their legs again or dorsiflex the foot in walking, owing entirely to neglect of the muscles and joints, and constant straightening and correcting by active and passive movements. Injuries to joints, even though septic, are not a bar to movement; the joints should be gently moved and encouraged, short of pain, and it is wonderful what results will ultimately be obtained, provided attention is paid daily to movement (passive at tirst and later active and steadily increased).

As to pain, the attitude of most present-day surgeons is wrong. For the last twelve years I have treated all frac. tures of the scapula, clavicle, liumerus, and many of the forearm, withont splints. I have no hesitation in applying splints if I think they are needed, but after the thircl or fourth day patients are satisfied with the lie of their arms in a sling. 'The main point, however, is that every movement of all joints and muscles should be encouraged, first of all passively and as soon as possible actively, and this should be carried out frequently during the day. It is sur. prising to see a patient with a fractured humerus, with possibly overlapping, extending the arm, flexing it and lifting it from the side, in the course of a fortnight or three weelss; by the time the fracture has consolidated all the movements of the joints are perfect, provided the damage has not been too great. In simple fractures the results are admirable; in septic compound fractures I can say the same; but.watch must be kept for collections of pus about necrotic bone, and these must be opened and drained, but should never interfere with the passive and active movements of the part. I feel compelled to write this, because 1 have seen such bad results not only from hospitals abroad but from hospitals in England and scotland. A few examples of many that have passed through my hands may perhaps help to impress my view.

(a) Through-and-throngh bullet wound of the hamstring, with flexion of the knee, kept in the position of flexion for several weeks before coming under my care. Twenty to thirty pounds pressure over the extended knee cannot overcome the contraction, and the man is a probable cripple for life, walking on his toes with bent knee.

(b) Patient with a wound of the outer side of the humerus not completely fracturing the bone, kept in a right angle splint for eight weeks. Unable to flex and extend elbow. rigidity about the shoulder, unable to satisfactorily close the haud; now, after four months of massacse, unable to straighten the arm or flex it completely.

(c) Patient with a suppurating prepatella bursa kept for three weeks in a posterior splint and three weeks in a Thomas splint. Now scarcely any flexion of the knee.

(d) Patient with a suppurating and ankylosing knee-joint several months in splints. No movement in the plantar flexed toes, plantar flexion of the foot, and no dorsitlexion of the foot possible.

(e) Numerous fractures of the homerus kept three and four months in a right-angle splint, hecause there was a septic wound leading to bone. Practically no movement at shoulder, elbow, wrist, and, in some cases, fingers.

I could go on giving endless cases of a similar nature. This needless disablement of men is really the most dis. heartening thing I know in the war. Sir Auckland Geddes as a medical man, and certainly the Minister of Pensions, should look into the matter with care, for it is one which involves the man power of the nation. I feel absolutely certain that the future will see a great reaction from this most remarkable age of splints.

\section{VARIATIONS IN THE ACTIVITY OF THE CILIARY MUSCLES.}

BY

\section{MAJOR G. F. ALEXANDER, R.A.M.C.}

THE activity of the ciliary muscles can be realized only by the routine practice of estimating the refraction when they have been completely paralysed, and again after they have recovered from the effects of the cycloplegic. Complete paralysis being litherto only obtainable by the use of atropin, has been mainly restricted to children on account of the inconvenience of the prolonged paralysis of accommodation and the danger of inducing glaucoma in the elderly, and thus, as a 2 per cent. solution of homatropiu and cocaine has in the case of young and vigorous adults frequently only a partial paralysing effect, variations in the activity of the ciliary muscles in adults have largely escaped observation. Early in the year, however, I found that cycloplegia as complete as that obtained by atropin could be obtained by a saturated solution of homatropin (16. per cent.); a large drop may be instilled in each eye without fear of toxaemia, and recovery is as rapid as after. the use of the 2 per cent. solution-that is, in twenty-four to thirty-six hours-or if a drop of 1 per cent. eserin is instilled in two to three hours, and thus the benefit of atropin is obtained without the drawback of its prolonged paralysing effect. By the use of this solution in over 1,000 cases, supplemented by that of a special astigmatic chart, I have been able to observe some interesting variations in the activity of the ciliary muscles, which with those alreacly familiar are briefly summarized as follows:

1. Both in hypermetropia and myopia the tonicity of the ciliary muscles varies from time to time: the difference in the vision recorded by different observers or the same observer on different days is thus accounted for, increase of activity necessarily lessening hypermetropia and in creasing myopia, and relaxation the reverse.

2. In anisometropia unequal activity of the ciliary muscles frequently neutralizes more or less of the disparity.

3. Apart from anisometropia the ciliary muscles often show unequal tonicity, as is proved-(a) in either hypermetropia or myopia by difference in the corrections of the two eyes found on post-cycloplegic testing, although paralysis of the muscles showed that the eyes were equaliy ametropic; (b) in either hypermetropia or myopia, and even occasionally in emmetropia, by spasm of the accon. modation taking place to an unequal degree in the two eyes, and even occasionally in one eye only.

4. Spasm of accommodation, apart from its commonest causes-namely, astigmatism and exophoria, the latter naturally acting chiefly in myopia-is frequent from overuse of the eyes for near work, especially if tine, under the electric light, or in insufficient light: though naturally most marked in hypermetropia it is found also in myopia and emmetropia; it is observed chiefly in young and neurotic subjects, but is found also in others, and even when they are well beyond the presbyopic age (I have notes of one case of spasm in an enmetrope aged 48 year's). 
5. While the contraction of the fibres of the ciliary sphincter in the states above noted takes place uniformly all round, establishing a degree of general tonicity, a contraction of some of the fibres or their relaxation from fatigue relatively to the rest may take place in segments of the sphincter, and this may be-

A. Meridianal-that is, when the segments are at the opposite ends of a meridian of the lens, in which case contraction increases and relaxation diminishes the re. fractive power of the lens in this meridian only. Such variations in activity occurring in both hypermetropia or myopia may either-

(a) Correct regular astigmatism, contraction doing so when acting in the meridian of least curvature of the corvea and relaxation when acting in the meridian of greatest curvature of the cornea. In this way hypermetropic astigmatism may be corrected, and though this is stated by Eruest Clarke and others to be possible only up to $0.75 \mathrm{D}$, I liave recorded numerous cases of 2 and $3 \mathrm{D}$, two of $4 \mathrm{D}$, and two of $5 \mathrm{D}$ in this way fully corrected, an interesting feature of some of them being that this correction was unattended by any symptom of eyestrain, this applying to both cases of $4 \mathrm{D}$ noted. As on post-cycloplegic testing the cylinders found to correct the astigmatism in the static refraction were in some cases rejected, in others accepted, and in still others weaker ones were accepted, it is evident that, just as in the case of hypermetropia, we have to cousider manifest, latent, and total astigmatism, the amounts of which vary in different cases and are subject to variation in the same case. Auto-correction of a low degree of astigmatism is common long after the presbyopic age, and I lave notes of the correction of simple hypermetropic astigmatism of $0.75 \mathrm{D}$ at 58 years.

(b) Cause regular astigmatism, in which case the segments may vary from time to time, necessitating corresponding changes in the axis of the correcting cylinder.

B. Non-meridianal-that is, not corresponding to one of the chief meridians of the lens, when its refraction is increased eccentrically so that, either in the existence of corneal astigmatism or apart from it, irregular astigmatism -that is, not correctable by glasses-results with occasionally monocular diplopia.

6. At times, from the above causes-and this I have found up to the age of 58-one or both of the ciliary muscles may pass into a phase of "irritability" from which regular astigmatism nay be corrected or caused at intervals, and, if the latter, the meridian may be varied from day to day, while in either case the astigmatism may every now and then be rendered irregular, and then, if monocular diplopia is present, from variations in the segments the fainter object may appear displaced from the more distinct in different directions at different times Naturally the above abnormalities in the activity of the ciliary nuscles are a fertile source of asthenopia and are of special interest when found at an age at which they liave been for the most part unsuspected.

7. As to treatment, it becomes imperative in all cases of asthenopia in which spasm or irritability of the ciliary muscles may be the possible cause, apart from lessening as much as possible the near work and doing it in a good light screened from the eyes by a green shade, etc., to paralyse the ciliary muscles as above and estimate the static refraction; and if the cylinders found necessary are not accepted and weaker ones accepted are still productive of asthenopia and we do not fear glaucoma, to keep the accommodation thoroughly paralysed by atropin for several weeks while the cylinders correcting the total astigmatism are worn and general and nervous tonjc treatment carried out, when they will almost invariably be accepted-an alternative plan being to prescribe the cylinders correcting the total astigmatism for constant use, trusting to the corrective partial contraction of the cilia:y muscles giving way in time.

ST. ANDREW's COLLEGE, Toronto, is being converted into a military hospital, to serve as the chief demobilization centre for the whole of Canada. Twenty.five new buildings are to be erected, and when completed the institution will are to be erected, and when completed the institution will
cover twenty-flve acres of ground. Though not intended to be permanent, the hospital will be used for many years after the war for the treatment of invalided or incapaeitated soldiers.

\section{BLOOD PRESSURE IN PULMONARY TUBERCULOSIS.}

\author{
BY
}

RICHARD J. CYRIAX, M.D.BRUX., M.R.C.S., D.P.H.,

ASSISTANT RESIDENT MEDICAL OFFICER, MOUNT VERNON HOSPITAL, NORTHWOOD.

Is the Jocrisal of August 10th appeared an article by D1. E. F. Cyriax on blood pressure in unilateral war traumatisms, pointing out that the pressures in the two arms do not always coincide, and, furthermore, that the pressure may be higher in one arm than in the other on one day, and the exact opposite a few days later. He pointed out also that the actual readings were influenced neither by the arm from which the first reading was taken, nor by the interval between the two readings.

A somewhat similar phenomenon appears to occur in some cases of phthisis, whether unilateral or bilateralnamely, that the pressure in the two arms is by no means invariably the same, a source of serious error being thereby introduced into the whole question of blood pressure in this disease.

The following observations were made upon 19 cases under the care of Dr. T. N. Kelynack at the Mount Vernon Hospital, Northwood. The readings were taken at one sitting, first on one arm and then upon the other, as rapidly as was compatible with accuracy, with a Riva-Rocci instrument, by the auscultation method combined with palpation of the radial pulse. Both systolic and diastolic readings were taken, but it is especially to the former that I wish to direct attention at present. Differences of $5 \mathrm{~mm}$. or less have been disregarded in order to make every allowance for the rise induced by the constriction of the armlet.

Five patients, all males, were confined to bed, except where otherwise stated. Tubercle bacilli have been found in all their sputa. In two cases differences of more than $5 \mathrm{~mm}$. in the systolic pressures were not found; two ob. servations were made on each patient at intervals of some days. Both have lesions in both lungs.

R. B., aged 18. Inflitration right upper and apex right lower Septemoptysis 6 ounces September 18th

September 27th. Blood pressure: R. 121.68; J. 119.68.

(Systolic difference, $\mathbf{R} .+2=0$.)

October 4th. R. 136.83; L. 132.84. (R. $+4=0$ )

Sodium nitrite, one grain, administered four times daily.

October 9th. R. 115.79; L. 113.78. (R. $+2=0$.)

October 11th. R. (taken first) 118.94; L. 132.78. (R. - 14.)

October 12th. R. 114.82; L. (taken first) 131.80. (R. - 17.)

The systolic pressure is thus lowest at present upon the diseased side. Sodium nitrite is still being administered.

\section{CASE IV.}

W. J. B., aged 41 . Infiltration both upper lobes, principally on the left.

September 27th. R. 124.84 ; L. 120.84. (I. $-4=0$.)

October 7th. Dr. Kelynack considered that a fresh spread was probably taking plaee. October 9th. Dr. Kinton noticed hat the principal activity was on the left side.

October 10th. R. (taken first) 102.85; L. 116.80. (L. + 14.)

October 12th. R. 124.88 ; L. (taken first) 136.90. (L. + 12.)

The systolic pressure is thus higher upon the side which is most active at present.

H. J. W., aged 47. Infiltration both upper lobes, principally upon the left, scattered foci throughout.

October 2nd. R. 105.80; L. 101.80. (L. $-4=0$.)

October 4th, 5th, 6th. Patient rested up two hours.

October 7 th. Sputum stained. Rest in.bed.

October 10th. R. 128.84; L. (taken first) 114.82. (L. - 14.)

October 12th. Patient rested up two hours. 'I'wo hour after return to bed, R. (taken first) 116.82; I. 109.83. (L. - 7.) All the above readings were taken while the patients were n bed.

In the remaining 14 cases, not confined to bed, differences in the systolic pressure of more than $5 \mathrm{~mm}$. were found in 6 . All six are cases of bilateral disease; tubercle bacilli have been found in all their sputa. The differences amounted, in the male patients, to 7,8 , and $13 \mathrm{~mm}$. respectively; in the female patients to 14,8 , and $10 \mathrm{~mm}$

I am convinced that the differences cannot be die to the constriction of the armlet. If they were so due, the same relation'slip ought invariabiy to subsist between the reading taken from the first and that taken from the second arm. No such relationship has been found. 